\title{
Dosage des bases puriques et pyrimidiques par chromatographie liquide à haute performance
}

\author{
B Lassalas, JP Jouany, L Broudiscou
}

INRA, Centre de Clermont-Ferrand-Theix, 63122 Saint-Genès-Champanelle, France

\begin{abstract}
Summary - High performance liquid chromatographic determination of purine and pyrimidine bases. In order to estimate the flow of microbial proteins entering the small intestine in ruminants, reversed phase high performance liquid chromatography (HPLC) was applied to separate purine and pyrimidine bases for $12 \mathrm{~min}$. This method was used to analyse duodenal digesta and microbial pellets isolated from the rumen contents.
\end{abstract}

L'estimation de la biomasse microbienne et de la quantité de protéines qu'elle renferme présente un intérêt particulier dans les études de digestion et de nutrition des ruminants. Cette mesure n'a pu être faite jusque là que de manière indirecte, en utilisant des marqueurs dits spécifiques des micro-organismes que l'on veut étudier. Très tôt, les chercheurs ont pensé que les acides nucléiques (AN) répondaient à ce critère (Gausserès et Fauconneau, 1965) si l'on admet comme Mac Allan et Smith (1973) que la totalité des AN provenant des aliments est dégradée dans le rumen. La présence de ces acides dans le duodénum est donc directement reliée à celles des micro-organismes. Outre les nombreuses critiques dont ils sont l'objet en tant que marqueurs microbiens, les AN ont été peu utilisés essentiellement à cause de la lourdeur des méthodes de dosage. Ushida et al (1985) ont proposé une méthode simplifée qui permet de doser les AN en mesurant l'absorbance à $260 \mathrm{~nm}$ des bases puriques qui ont été préablement libérées et isolées. Nous proposons ici une méthode de dosage des bases puriques et pyrimidiques par HPLC, qui présente les avantages d'être plus spécifique, plus rapide et plus précise que le dosage global des AN par spectrophotométrie.

Matériel et méthodes - 1) Libération des bases puriques et pyrimidiques par une hydrolyse oxydante : les échantillons (lyophilisat microbien ou contenu digestif) sont broyés finement $(0,8 \mathrm{~mm})$. On mélange $0,5 \mathrm{~g}$ d'échantillon à $3 \mathrm{ml}$ d'acide perchlorique $\mathrm{HClO} 4$ à $70 \%$ dans un tube à essai à vis de $30 \mathrm{ml}$ (Sovirel). Le tube hermétiquement clos est introduit dans un bainmarie à $95^{\circ} \mathrm{C}$ pendant $1 \mathrm{~h}$. Après refroidissement, $17,5 \mathrm{ml}$ de tampon dihydrogénophosphate d'ammonium NH4 H2 $\mathrm{PO} 40,0285 \mathrm{M}$ sont ajoutés au contenu du tube qui est ensuite placé pendant $15 \mathrm{~min}$ à $95^{\circ} \mathrm{C}$. La solution refroidie est filtrée sous vide sur une membrane en nitrate de cellulose de $8 \mu \mathrm{m}$. Les filtrats peuvent être congelés avant leur analyse.

2) Analyse des bases par HPLC : la colonne chromatographique $(4,6 \mathrm{~mm}$ de diamètre, 22 $\mathrm{cm}$ de longueur $+3 \mathrm{~cm}$ d'une précolonne identique à la colonne et reliée à celle-ci sans volume mort) est remplie d'une phase inverse $\mathrm{C} 18$ de $5 \mu \mathrm{m}$. La phase mobile est constituée d'un gradient binaire de tampon acétate de potassium ( $50 \mathrm{mM}, \mathrm{pH} \mathrm{6}$ ) et de méthanol (tableau I) qui sont dégazés en continu avec He. Les bases sont détectées en sortie de colonne par spectrophotométrie à $254 \mathrm{~nm}$ (fig 1). Le signal est traité par un intégrateur-calculateur. La durée d'une analyse est de l'ordre de $12 \mathrm{~min}$ ( +8 à $10 \mathrm{~min}$ pour retourner aux conditions initiales).

3) Étalonnage de la colonne : les solutions étalons contenant les bases pures sont préparées avec les mêmes solvants que les échantillons ( $3 \mathrm{ml} \mathrm{HClO} 4+17,5 \mathrm{ml}$ tampon $\mathrm{NH}_{4} \mathrm{H}_{2}$ PO4). Une droite d'étalonnage répondant à l'équation $y=a x+b(y=$ aire du pic correspondant, $x=$ concentration de la base) est établie pour chaque base.

Résultats et discussion - Le dosage direct dans l'hydrolysat évite les étapes de 
complexation des bases puriques par un sel d'argent, de lavage du précipité et de sa solubilisation avant la lecture au spectrophotomètre, qui entraînent des pertes non reproductibles de produits et nuisent à la validité de la méthode de Ushida et al (1985). En outre, d'autres substances absorbant à $254 \mathrm{~nm}$, peuvent être présentes dans la solution et interférer dans le dosage global des AN. Les différences notées dans les coefficients d'absorption de chaque base posent le problème du choix des AN comme standard dans la méthode globale. Outre sa spécificité, la technique HPLC permet de connaître la composition qualitative et quantitative des $A N$, ce qui ouvre des perspectives pour l'identification de la biomasse microbienne à partir d'une méthode basée sur le profil des bases des grands types microbiens.

La méthode de dosage proposée est largement inspirée de celle décrite par Schweinsberg et Ti Li Loo (1980). Nous avons modifié la phase mobile en remplaçant le tampon phosphate qui se cristallise dans les têtes de pompes par un tampon acétate. La composition du gradient a

Tableau I. Composition du gradient de la phase mobile (\%).

\begin{tabular}{llrl}
\hline Temps (min) & $\begin{array}{l}\text { Tampon } \\
\text { acétate }\end{array}$ & Méthanol & $\begin{array}{c}\text { Débit } \\
(\mathrm{m} / \mathrm{min})\end{array}$ \\
\hline 0 & 94 & 6 & 1 \\
7 à 10 & 85 & 15 & 1 \\
\hline
\end{tabular}

aussi été modifiée de façon à réduire le temps d'analyse.

La méthode est répétable puisque le coefficient de variation calculé pour la somme des bases, à partir d'un échantillon injecté 7 fois de suite, varie de 1,0\% pour I'ARN de levure et le contenu duodénal, à $1,9 \%$ pour un échantillon de bactéries libres du rumen. Toutefois, le coefficient de variation diffère suivant les bases pour un même échantillon (tableau II). La quantité minimale mesurable est de $5 \mathrm{nmol} / \mathrm{ml}$ pour la cytosine, l'uracyle, la guanine, la thymine, et de $30 \mathrm{nmol} / \mathrm{ml}$ pour l'adénine.

Gausserès B, Fauconneau G (1965) Ann Biol Anim Biochim Biophys 5, 5-20

Mac Allan AB, Smith RH (1973) Br J Nutr 29, 467-474

Schweinsberg PD, Ti Li Loo (1980) J Chromatogr 181, 103-107

Ushida K, Lassalas B, Jouany JP (1985) Reprod Nutr Dév 25, 1037-1046

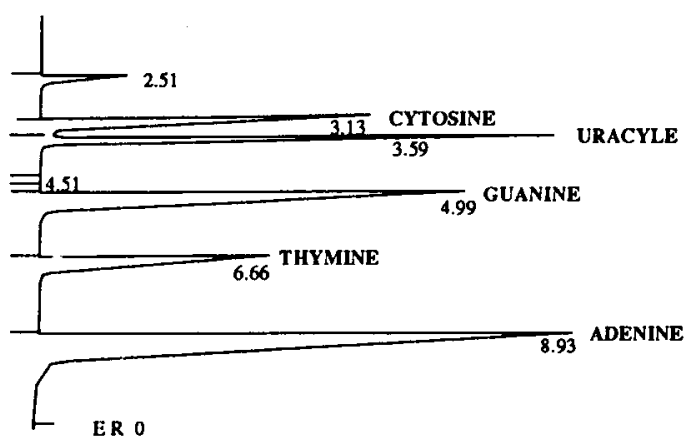

Fig 1. Chromatogramme d'un mélange de bases pures.

Tableau II. Coefficients de variation (\%) de chacune des bases présentes dans les 3 types d'échantillons.

\begin{tabular}{lcccccc} 
Échantillons & Cytosine & Uracyle & Guanine & Thymine & Adénine & Somme des bases \\
\hline Bactéries du rumen & 0,80 & 1,02 & 4,70 & 6,15 & 4,40 & 1,90 \\
Contenu de duodénum & 0,80 & 0,94 & 1,35 & 0,90 & 2,12 & 0,92 \\
ARN de levure & 0,90 & 3,25 & 0,50 & - & 1,10 & 1,00 \\
\hline
\end{tabular}

